\title{
Publish by Example
}

\author{
Sonia Guéhis \\ University of Paris Dauphine \\ sonia.guehis@lamsade.dauphine.fr \\ David Gross-Amblard \\ University of Bourgogne \\ david.gross-amblard@u-bourgogne.fr \\ Philippe Rigaux \\ University of Paris Dauphine and INRIA-Orsay \\ philippe.rigaux@lamasde.dauphine.fr
}

\begin{abstract}
We propose an approach for producing database publishing programs by example. The main idea is to interactively build an example document, representative of the program output. The system infers from this document, without ambiguity, the publishing program. The end-user does not need to know a programming language, a query language or the database schema.
\end{abstract}

\section{Introduction}

We consider the problem of producing "dynamic" documents that contain data retrieved from a relational database. We impose no restriction on our concept of document: it can be non-structured character data (e.g., an email), an XML document (for data exchange purposes), an HTML document (web site publishing), a IATEX file or an Excel spreadsheet, etc. Their common characteristic is to consist both of static parts and dynamic parts, the latter being values extracted from the database when the document is produced. We call database publishing the process of creating dynamic documents from a relational instance. The most typical example is the production of (X)HTML pages in dynamic web sites. We use it for illustration purposes in this paper.

Relational database publishing is technically simple, but requires in practice the association of programming tools and database concepts which often make the production tedious and error-prone. It constitutes in particular an intricate practical aspect of web site engineering [10]. Specialized languages, such as Servlets/JSP, PHP or ColdFusion [3], bring partially satisfying solutions. However, in all cases, writing a database publishing program requires heterogeneous technical knowledge, including: (i) the basics of a programming language (say, Java/JSP); (ii) a query language (say, SQL); (iii) the database schema.

In the present paper we propose a simple mechanism to produce database publishing programs. The main idea is to interactively construct a sample dynamic document which can then be used to infer without ambiguity the publishing program. What makes such an approach effective is the inherent simplicity of relational publishing which does not require the full power of general-purpose programming and query languages.

The benefits are twofold. First the proposed mechanism does not require any technical expertise. As such if offers to non-expert users an opportunity to create rich documents with minimal efforts. Second it constitutes a generic approach which holds independently from a specific environment, does not require any preliminary decision regarding programming practices and conventions, and avoids the tedious and repetitive programming tasks. One obtains a high-level specification of publishing programs, with potential support for software engineering tasks (e.g., verification) as well as database optimization.

\section{Overview of the approach.}

Figure 1 presents the main components of our approach, and their respective roles in a publishing system. First, we formalize relational database publishing as a "document query language", called DocQL, already proposed in preliminary form in [6]. A DocQL query can be seen as a syntax-neutral (declarative) specification of a publishing program written in Java/JSP or in any equivalent programming framework. Producing a DocQL query constitutes the target of the publish-by-example process.

The publishing model relies on the concepts of canonical documents and canonical instances. A canonical document characterizes uniquely a DocQL query $q$, and therefore the publishing program which can be derived from $q$. The user interacts with a WYSIWYG graphical editor which lets him construct a canonical document $D$ from a canonical instance $I_{C}$. 


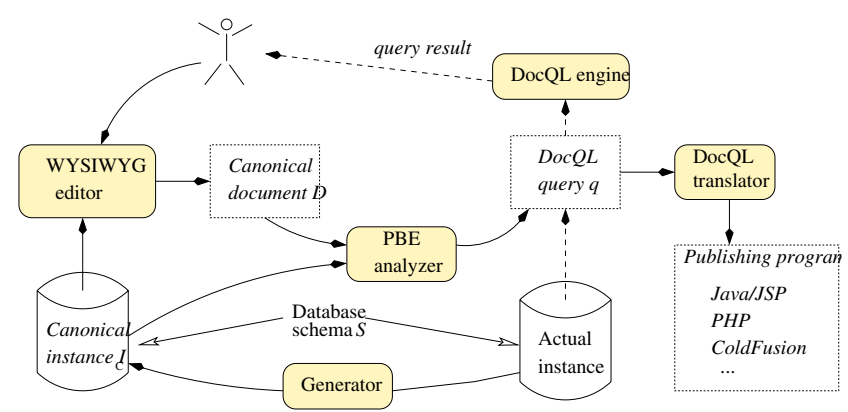

Figure 1. Overview of the publish by example process

A canonical instance $I_{C}$ of a schema $S$ is an instance such that, for any DocQL query $q$, there exists a canonical document over $I_{C}$ that characterizes $q$. Proposing a canonical instance to a user is tantamount to the ability of producing, by example, all the possible DocQL queries that can be expressed over $S$. The canonical instance is a predetermined instance of the schema $S$, generated by the system administrator using an instance generator.

Given a canonical instance $I_{C}$ and a canonical document $D$ built over $I_{C}$, the Publish By Example analyser infers a unique DocQL query $q$. The user can then either run $q$ over the actual instance, through the DocQL engine, or translate $q$ to a traditional publishing program, written in any convenient language.

\section{Running example.}

Throughout the paper we illustrate our approach over a sample movie database representing movies with their (unique) director and their (many) actors. Figure 2 shows a simple database instance. Primary keys are in bold, and foreign keys in italic.

In the rest of this paper, Section 2 briefly introduces the DocQL language. We then describe the publication model in Section 3.Section 4 positions our proposal with respect to the state of the art, and Section 5 concludes the paper. A long version of the paper, along with an on-line demonstrator, is available at http://www.lamsade.dauphine.fr/rigaux/docql.

\section{The publishing language DocQL}

We give the main features of the publishing query language DocQL. Since this does not constitute a contribution of the present paper, we limit the presentation to a few illustrative examples. Formal definitions can be found in [6].

\section{Data model.}

DocQL aims at a concise specification of publishing

\begin{tabular}{l|c|c|l} 
title & year & id_director & genre \\
\hline Unforgiven & 1992 & 20 & Western \\
Van Gogh & 1990 & 29 & Drama \\
Kagemusha & 1980 & 68 & Drama \\
Absolute Power & 1997 & 20 & Crime \\
\hline \multicolumn{3}{|l}{ Movie } \\
id & last_name & first_name \\
\hline 20 & Eastwood & Clint \\
21 & Hackman & Gene \\
29 & Pialat & Maurice \\
30 & Dutronc & Jacques \\
68 & Kurosawa & Akira \\
\hline \multicolumn{3}{c}{ Artist }
\end{tabular}

\begin{tabular}{l|l|l} 
title & id_actor & character \\
\hline Unforgiven & 20 & William Munny \\
Unforgiven & 21 & Little Bill Dagget \\
Van Gogh & 30 & Van Gogh \\
Absolute Power & 21 & President Allen Richmond \\
\hline \multicolumn{2}{|c}{ Cast }
\end{tabular}

Figure 2. An instance of the Movies database

programs. The language captures with a uniform and simple syntax the queries and programming instructions used to build dynamic documents. It relies on a navigation mechanism in an instance $I$ modeled as a labeled directed graph $\mathcal{G}_{I}$. Tuples are seen as internal nodes, values as leaf nodes, and edges represent either tuple-to-tuple relationships or tuple-to-attribute dependencies.

Figure 3 shows the data graph of the instance of Figure 2. We distinguish functional dependencies between nodes (e.g., between a movie node and its director node) and multivalued dependencies (e.g., between a movie node and its actor nodes). The former are shown with white-headed arrows, the latter with black ones.

If $N_{1}$ and $N_{2}$ are two nodes in the data graph, we note $N_{1} \stackrel{p}{\rightarrow} N_{2}$ if $N_{2}$ functionally depends on $N_{1}$, and we say that $p$ is a unique path. For instance if $N_{1}$ is a movie and $N_{2}$ the last name of its director, then $N_{1}$ director.last_name $N_{2}$, and director. last_name is a unique path. Else we note $N_{1} \stackrel{p}{\rightarrow} N_{2}$ and say that $p$ is an instance of a multiple path.

The context of a node $N$ is the set of leaf nodes that functionally depend on $N$. The neighborhood of $N$ is the set of nodes $N^{\prime}$ such that there exists an elementary multiple path (i.e., with only one edge) $N \stackrel{p}{\rightarrow} N^{\prime}$. Consider again Figure 3 and the node (of type Movie) in the box. Its context consists of the values Unforgiven (title of the movie), 1992 (year), Western (genre), 20, Clint, and Eastwood (resp. the id, first name and last name of the director who is uniquely determined by the movie). The neighborhood consists of the two nodes Cast.

\section{Query language.}

DoCQL combines navigation in the data graph with in- 


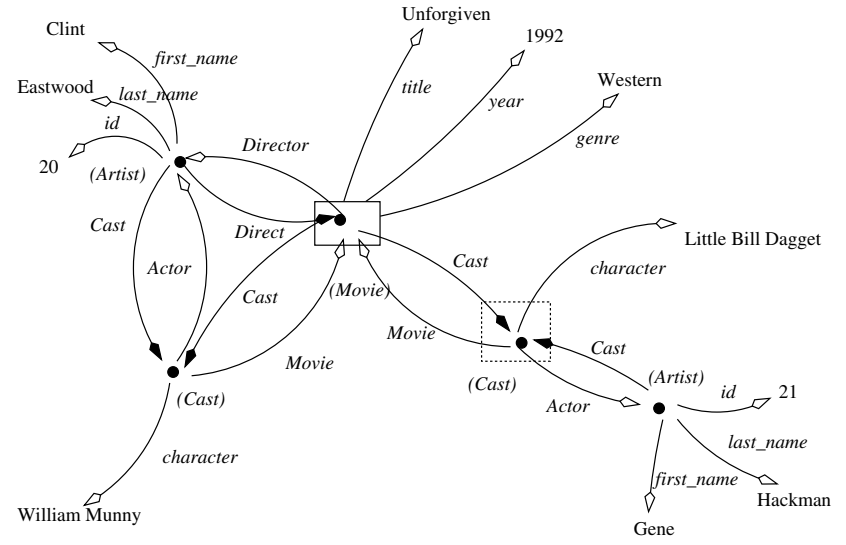

Figure 3. A subset of the data graph of our sample instance

stantiation of the textual fragments that contribute to the final document. A DocQL query is essentially a tree of path expressions which denote the part of the graph that must be visited in order to retrieve the data to include in the final document. Path expressions use an XPath-like syntax. An expression $p$ is interpreted with respect to an initial node $N_{i}$ (unless it begins with db which plays the role of / in XPath), and delivers a set of nodes, called the terminal nodes of $p$ (with respect to $N_{i}$ ). Each path is associated to a fragment which is instantiated for each terminal node. Path and fragments are syntactically organized in rules of the form @path [condition] \{fragment\}, where path is a path expression, condition is a node condition and fragment is the fragment instantiated for each instance of path.

The following example shows a DocQL query over our Movies database. It produces a (rough) document showing the movie Unforgiven along with its director and actors.

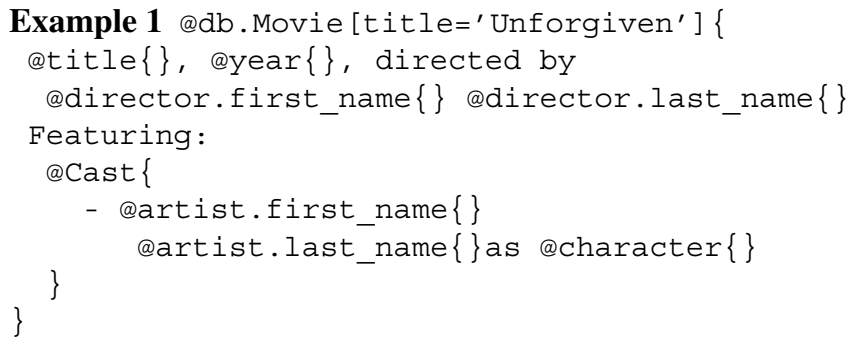

The semantics of the language corresponds to nested loops that explore the data graph, one loop per rule. This navigation produces the trace of a query $q$, which is a finite unfolding of the graph $\mathcal{G}_{I}$ representing the nodes visited during the evaluation of $q$. The result of a query is obtained by "decorating" the nodes of its trace with the (static) character data of their associated rules. Applied to the data graph of Fig- ure 3, one obtains the following document as result of the previous example:

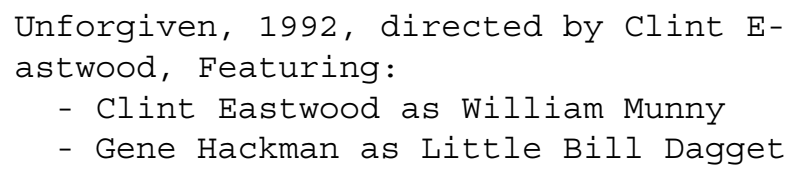

\section{The Publish by Example Model}

We now develop our model by defining our two key concepts: canonical documents and canonical instances.

\section{Structure of canonical documents.}

A canonical document has a hierarchical structure. Each node of the document's structure is called a block. A block is a character string with (optional) references to other blocks. The textual part of a block consists of fixed text and values from the active domain (i.e., leaves) of the graph $\mathcal{G}_{I}$.

Let $\Sigma$ be an alphabet. $\mathcal{F} \subset \Sigma^{*}$ denotes the set of static fragments, and dom $\subset \Sigma^{*}$ denotes the active domain of $\mathcal{G}_{I}$. For the sake of simplicity, we suppose that $\mathcal{F} \cap$ dom $=\emptyset$, in order to distinguish elements from theses two sets. In practice, the distinction may rely on a syntactical convention We also assume a set $\mathcal{B}$, distinct from the previous ones, of block identifiers.

Definition 1 (Block) $A$ block $B$ is a pair $(i, b)$, where $i \in$ $\mathcal{B}$ is the block identifier and $b \in(\mathcal{F}|\boldsymbol{d o m}| \mathcal{B})^{*}$ is the block body. We denote by components $(B)$ the set of blocks recursively referenced by the body of $B$.

We are interested in blocks that can be unambiguously interpreted with respect to $\mathcal{G}_{I}$. We first define the notion of representative node of a block.

Definition 2 (Representative node of a block) $A$ node $N \in \mathcal{G}_{I}$ is representative of a block $(i, b)$ if and only if each value $v \in$ dom in $b$ belongs to the context of $N$.

Recall that the context of a node $N$ is the set of values $v$ that functionally depend on $N$. Consider for example the block $B$ with body "Unforgiven, published in 1992 and directed by Clint Eastwood", where values from dom appear in bold. The node $N$ corresponding to the movie Unforgiven is representative of $B$, because each value $v$ belongs to the context of $N$ (see Figure 3).

Let $B$ be a block and $N$ be a representative node of $B$. We say that $B$ is valid with respect to $N$ if there exists a representative node for each component of $B$, such that the structure of the subgraph induced by these nodes is homomorphic to the structure of $B$. Formally:

Definition 3 (Block validity) $A$ block $B$ is valid with respect to a node $N$ if and only if $N$ is a representative node, 
and for each child block $B_{i}$ of $B$ there exists a node $N_{i}$ in the neighborhood of $N$ such that $B_{i}$ is valid with respect to $N_{i}$.

A block $B$ is said to be valid on $\mathcal{G}_{I}$ if there exists a node $N$ in $\mathcal{G}_{I}$, such that $B$ is valid with respect to $N$.

Consider block $B_{1}$ with body "Unforgiven, 1992, featuring: \#ref(2)", referencing block $B_{2}$ with body "Little Bill Dagget played by Gene Hackman". $B_{1}$ is valid with respect to the node $N_{1}$ (framed with solid lines in Figure 3 ) because we can find a node $N_{2}$ (framed with dotted lines), representative of $B_{2}$ in the neighborhood of $N_{1}$, with $N_{1} \stackrel{\text { Cast }}{\rightarrow} N_{2}$. Note that Little Bill Dagget, Gene and Hackman, all belong to the context of $N_{2}$.

\section{Interpretation of valid blocks.}

Given a block $B$ valid on $\mathcal{G}_{I}$, our goal is to define a mapping that uniquely determines a query $q$ from $B$ and $\mathcal{G}_{I}$. A complementary question is to know, given a query $q$, whether there exists a block $B$ valid on $\mathcal{G}_{I}$ that determines $q$. We introduce three constraints on $\mathcal{G}_{I}$ : completeness, minimality and non-ambiguity. An instance is said complete if, for each node $N$ of type $r \in \mathcal{R}$, and each edge type $e$ of the form $r \stackrel{a}{\rightarrow} r^{\prime}$, there exists at least one edge $N \stackrel{a}{\rightarrow} N^{\prime}$. The instance is minimal is there is at most one such edge. The non-ambiguity condition is defined as follows:

Definition 4 (Non-ambiguous instance) An instance $\mathcal{G}_{I}$ is non-ambiguous if and only if, for all node $N$, the following conditions hold:

- if $N^{\prime}$ is a node in the context (resp. in the neighborhood) of $N$, there exists only one path $p$ such that $N \stackrel{p}{\rightarrow} N^{\prime}$ (resp. $\left.N \stackrel{p}{\rightarrow} N^{\prime}\right)$;

- if $N_{1}$ and $N_{2}$ are two nodes of the neighborhood, then $\operatorname{context}\left(N_{1}\right) \cap \operatorname{context}\left(N_{2}\right)=\emptyset$.

Checking this property for a given instance is easily done by visiting each node and verifying its context and neighborhood.

The first condition requires that if $N^{\prime}$ is a node in the context or in the neighborhood of $N$, then the path leading from $N$ to $N^{\prime}$ can be uniquely determined. The instance on Figure 3 would be ambiguous if, for example, the movie title and the director's name were both 'Eastwood' (condition on the context).

The second condition ensures that a node in the neighborhood of $N$ can be uniquely determined by any value of its context. Still looking at Figure 3, assume that we add a (multiple) path producer between movies and artists. The instance becomes ambiguous if the producer's name is William Munny, since we can no longer determine whether this value is the character of the neighborhood's node Cast or the name of the neighborhood's node Producer.
The instance of Figure 3 is non-ambiguous, but not minimal nor complete. If we remove the node squared with dashed lines (and the corresponding Artist subgraph), the instance becomes also minimal (and complete).

If the instance is minimal and non-ambiguous, a unique tree of representative nodes can be associated to a valid block $B$, with one node for each descendant of $B$ and $B$ itself. Since $\mathcal{G}_{I}$ is minimal, this tree can be viewed as the trace of a query (i.e., the tree of nodes visited during query evaluation). Given a valid block $B$ and a data graph $\mathcal{G}_{I}$, we call generating queries the queries $q$ such that $B=q\left(\mathcal{G}_{I}\right)$. In general, two non-equivalent queries $q$ and $q^{\prime}$ may yield the same result on a specific instance $\mathcal{G}_{I}$. However, when $\mathcal{G}_{I}$ is a non-ambiguous instance, there exists a unique minimal element (up to equivalence) in the generating set of a block $B$. Minimality is defined with respect to query (and trace) containment.

Definition 5 (Minimal generating query) Let $B$ be $a$ valid block on an instance $\mathcal{G}_{I}$. The minimal generating query $q$ of $B$ is the smallest element (up to query equivalence) of the set of generating queries of $B$ according to query containment.

A syntactic expression of the minimal generating query can be built as follows. First, the tree $T$ of the representative nodes of $B$ in $\mathcal{G}_{I}$ is computed. One method to achieve this is to consider values from each block as keywords and to perform a search of representative nodes according to these keywords. A simpler approach is to gather information on the representative nodes visited by the user during the interactive construction of the block. The latter solution is applied in our prototype. Second, once the tree of representative nodes $T$ is obtained, the rules of the DocQL query are recursively built according to the following procedure:

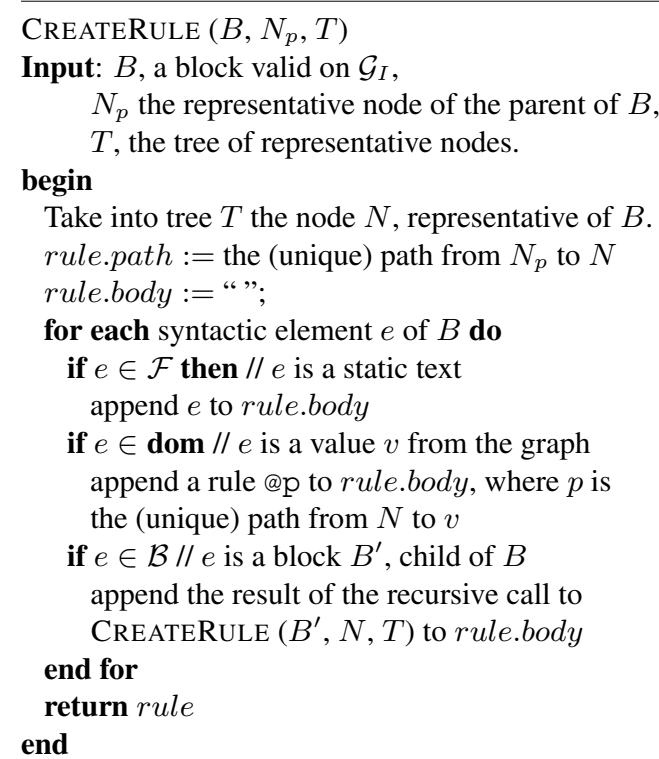


This procedure is initially called with the root block and the virtual node corresponding to the graph entry point. It is noteworthy that the soundness of this procedure is guaranteed only on a non-ambiguous instance.

This algorithm builds a DOCQL query without predicates. The structure of a valid block yields only the specification paths in the database, without the ability to express conditions on the encountered values. In order to complete this specification, the user (assisted by the system) may provide a function $f$ binding to each block $B$ a condition (or a conjunction of conditions). A condition on a block $B$ is defined by $a \theta b$, where $\theta$ is a relational comparison operator, and $a$ and $b$ are unique paths or simple values. We can finally define canonical documents:

Definition 6 (Canonical document) $A$ canonical document of a query $q$ is a pair $(B, f)$, where $B$ is a valid block such that $q$ is (equivalent to) the minimal generating query of $B$, and $f$ is a function that binds a conjunction of conditions to each component of $B$.

A Publish-By-Example interface must assist the interactive construction of a canonical document representing the awaited query $q$, in the most intuitive and simple way. Note that, in order to produce a canonical document characterizing $q$, all the representative tuples required for block interpretation must be available in the manipulated instance. The following section addresses this issue.

\section{Canonical instances.}

The construction of a canonical document $D$ assumes that the instance proposed to the user allows both the construction and the interpretation of $D$. This gives rise to the question of constructing a specific instance, called canonical instance, that allows to build a canonical document for all the possible queries over the graph schema.

Definition 7 (Canonical instance) $A n$ instance $\mathcal{G}_{I}$ of $a$ schema $S$ is a canonical instance if, for any query $q$ over $S$, there exists a canonical document of $q$ on $\mathcal{G}_{I}$.

An instance is canonical if it is complete, minimal and non-ambiguous. Completeness is required for allowing all the possible navigations in the graph with respect to the schema, whereas the minimality and non-ambiguity serve to a proper interpretation of a canonical document as a query. Recall that an instance is complete if, during the navigation in the graph, we can find at any moment a choice for each possible path type.

As an example, consider the relational instance of Figure 3, and assume that Movie contains only the tuple Kagemusha. Suppose that a user wants to produce a publishing query showing a movie with the list of its actors. It is not possible to build a canonical document for this query on this instance, since the casting is unkown for Kagemusha. This instance is not canonical.

If, instead of Kagemusha, Movie contains the tuple Van Gogh, we can produce the following canonical document that shows a film, its director and its actors:

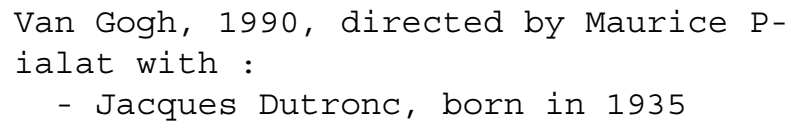

By contrast, the instance containing only film Van Gogh is not sufficient to build an example for a publishing query showing a film, its actors, and for each actor, the list of films possibly directed by this actor. Indeed, in this instance Jacques Dutronc is not a director. Nevertheless the relationship between an artist and a movie as a director exists, and a user may want to exploit this relationship. Therefore this instance is still not canonical.

Finally, as a last example, consider the instance of Figure 3 in which the only represented movie is Unforgiven. This instance allows the construction of the canonical document giving a film, its actors, and the films directed by these actors:

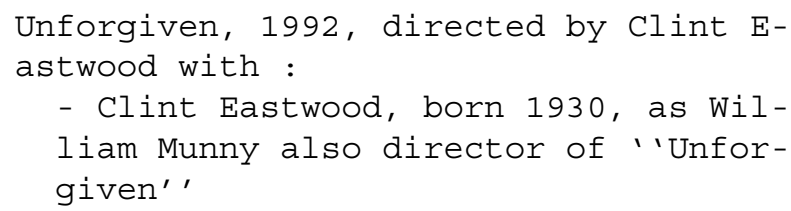

This document is possible thanks to a cycle into the data graph, instance of the cycle Movie $\rightarrow$ Director $\rightarrow$ Actor $\rightarrow$ Movie in the graph schema. The cycle size in the instance is proportional to the cycle size in the schema. With the two nodes Eastwood and Unforgiven, the instance cycle has a minimal size (two edges). Although satisfying with respect to the completeness of the canonical instance as a support for canonical documents, a shortcoming of a small cycle is to show repeatedly the same node at different places in a document, with a possible confusion on the role of each occurrence. In the previous example, Eastwood and Unforgiven both appear twice, each time in a different context. This may be misleading to the user, and results in an apparent lack of generality.

The instance can be extended to longer cycles of size $k \times n$, where $n$ is the cycle size in the graph schema and $k \geq 1$ is a parameter of the system. Figure 4.a shows a minimal cycle in our sample instance, and Figure 4.b its generalization to a cycle of length $k \times n$.

Observe that the occurrence of a cycle in the graph schema implies the occurrence of a cycle in the canonical instance, otherwise the instance would not be complete. In case of a path without cycle, the two extreme nodes would be left without "corresponding node", and the ability 


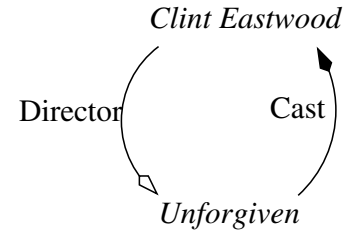

a. Minimal cycle (2 edges)

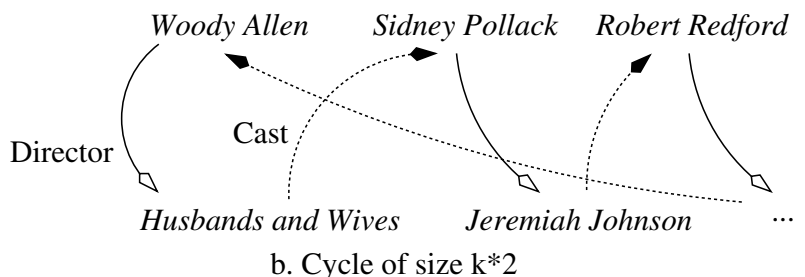

Figure 4. Cycle in a canonical instance

to build a canonical document from these nodes would be compromised.

The production of a canonical instance must ensure that the required properties are verified. If only cycles of minimal size are to be constructed, then the construction algorithm is straightforward: a node is instantiated for each node type of the schema, and an edge between these nodes is instantiated for each edge type in $E$. We describe in the following a more sophisticated algorithm that takes into account an expansion factor $k$ for cycle size.

The algorithm maintains a global array nodes $s_{r}$ for each node type $r$ of the schema. nodes $s_{r}$ contains the sequence of instances built by the algorithm, denoted nodes $_{r}[1]$, nodes $_{r}[2]$, etc. The algorithm returns a path $r_{1} \cdot e_{1} \cdot r_{2} \cdot e_{2} \cdot \cdots \cdot r_{n}, r_{i} \in V$ and $e_{i} \in E$, extended at each recursive call, and representing nodes and edges created during function calls. We use two auxiliary functions on paths:

1. $\operatorname{dist}(p a t h, r)$ returns the number of steps in path since the first occurrence of a node of type $r$;

2. $n b($ path,$r)$ returns the number of occurrences of a node of type $r$ in path;

The algorithm takes as input a node $N$, the type $e$ of the edge to create, and the path created since the initial call. The global variable $K$ denotes the minimal size required for a cycle.

CONSTRUCT $(N, e, p a t h)$

Input: $N \in V_{I}$, a node, $e$ an edge type such that begin

$N$ is an instance of initial $(e)$, path the path.

// We extract the type of the terminal node of $e$

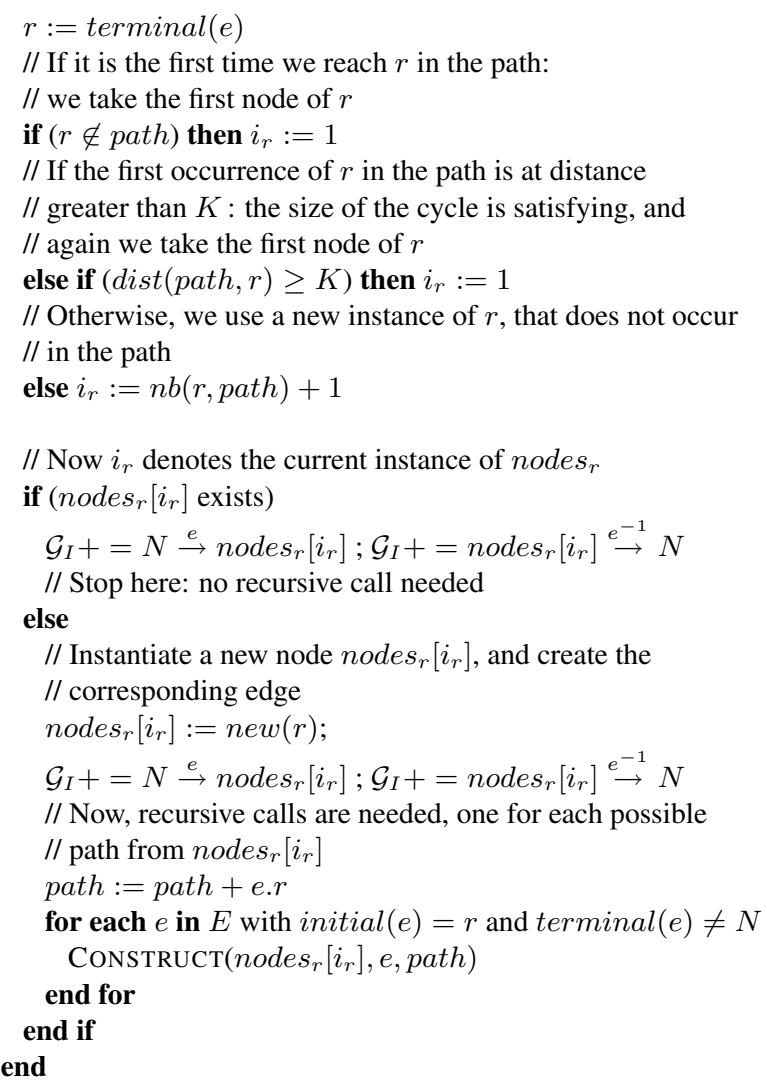

Algorithm ConstRUCT must be called for each connected component of the graph schema, taking any relation node type in each component as a starting point for the instance creation.

This algorithm builds a synthetic canonical instance, with somehow meaningless node values. In practice, relying on a real instance would yield more user-friendly node values. However there is no guarantee to find a canonical instance into a real instance. In that case it is necessary to complete the instance with synthetic values, or to link artificially existing but unrelated values.

We implemented a web-based editor and query system ${ }^{1}$ for our publication model. The system allows to build canonical documents, derives their associated DoCQL queries and may either immediately evaluate the query on a real instance, or save the query as a named dynamic fragment which can later on be composed with others.

\section{Related work}

Using graphical interfaces for expressing queries is an old concerns. The early language Query By Example

\footnotetext{
${ }^{1}$ Publicly accessible on the site $h t t p: / / w w w . l a m s a d e . d a u p h i n e . f r /$ rigaux/docql
} 
(QBE) [11] addresses the main principle of such visual tools: the query expression is based on an image of the result. QBE and its variants remain oriented toward the expression of relational queries, and deliver relational tables as result.

The "by example" paradigm has been adapted and extended to semi-structured data and XML document by many proposals: QSByE [4], QURSED[8] and XQBE [2]. QURSED is a web form and report generator, dedicated to the querying of semi-structured data. XQBE proposes an interface to automatically generate XQuery queries. All these tools help users to construct complex queries over directed labeled trees. Queries are displayed with a graph-based representationIn contrast, in our approach, the user does not manipulate a query but a query result. This limits the technical knowledge required from the user, and favors the integration of our tool with document editors.

An implementation of our model could take advantage of keyword-search techniques in relational database $[5,9,7]$. Applied to a canonical instance, they could probably deliver a non-ambiguous graph of representative nodes/tuples. This supports our belief that an interface based on alternative design principles is possible.

The publishing language which constitutes the target of our publishing process can be related to XML publishing. The specification of the exported data is usually expressed as a tree of co-related SQL queries and can be viewed as an abstraction of nested cursors over result sets. This is quite similar to the publishing mechanism adopted in the present paper. One can therefore envisage to adapt our examplebased approach to XML publishing languages.

Finally we note that our data model is closely related to the field of functional dependencies. In particular the concept of canonical instance shares with Armstrong relations its motivation of building a representative instance to assist the end-user in his designing tasks (see, in particular, [1]). Although we could have used this standard framework in a more direct way, we believe that the tailored approach chosen in the current paper fits more intuitively to our goals. In particular the graph-based representation is much more intuitive to the non-expert user than the scattering of information in relational tables.

\section{Conclusion}

We propose in this paper a simple and intuitive method for producing publishing programs. We described the formel model which states the main concepts and specially those of canonical documents and canonical instances.

Our publish-by-example mechanism is implemented and we are currently validating our tool with respect to an actual data-intensive web application (namely the MYREVIEW system, http://myreview.lri.fr) to check its ability to produce and maintain the set of dynamic fragments that constitute the view (presentation) part.

\section{References}

[1] C. Beeri, M. Dowd, R. Fagin, and R. Statman. On the Structure of Armstrong Relations for Functional Dependencies. J. ACM, 31:30-46, 1984.

[2] D. Braga, A. Campi, and S. Ceri. XQBE : A Visual Interface to the Standard XML Language. ACM Trans. on Database Systems, 30:398-443, 2005.

[3] Macromedia ColdFusion MX 7, 2007. http://www.adobe.com/fr/products/coldfusion/.

[4] Irna M. R. Evangelista Filha, Alberto H. F. Laender, and Altigran Soares da Silva. Querying Semistructured Data By Example: The QSByE Interface. In Workshop on Information Integration on the Web, pages 156-163, 2001.

[5] G.Bhalotia, C.Nakhe, A.Hulgeri, S.Chakrabarti, and S. Sudarshan. Keyword Searching and Browsing in databases using BANKS. In Proc. IEEE Intl. Conf. on Data Engineering (ICDE), pages 431-440, Washington, USA, 2002. IEEE Computer Society.

[6] S. Guéhis, P. Rigaux, and E. Waller. Data-driven Publication of Relational Databases. In Proc. IEEE Intl. Database Engineering \& Applications Symposium (IDEAS'06), 2006. Also in BDA'06.

[7] V. Hristidis and Y. Papakonstantinou. DISCOVER: Keyword Search in Relational Databases. In Proc. Intl. Conf. on Very Large Data Bases (VLDB), pages 670-681, Hong Kong, China, 2002.

[8] Y. Papakonstantinou, M. Petropoulos, and V. Vassalos. QURSED: Querying and Reporting Semistructured Data. In Proc. ACM SIGMOD Symp. on the Management of Data, 2002.

[9] S.Agrawal, S.Chaudhuri, and G.Das. Dbxplorer: A system for keyword-based search over relational databases. In Proc. IEEE Intl. Conf. on Data Engineering (ICDE), pages 005-016, Los Alamitos, CA, USA, 2002.

[10] S.Ceri, P.Fraternali, A.Bongio, M.Brambilla, S.Comai, and M.Matera. Designing Data-Intensive Web Applications. Morgan-Kaufmann, 2002.

[11] Moshé M. Zloof. Query-by-example: A data base language. IBM Systems Journal, 16(4):324-343, 1977. 